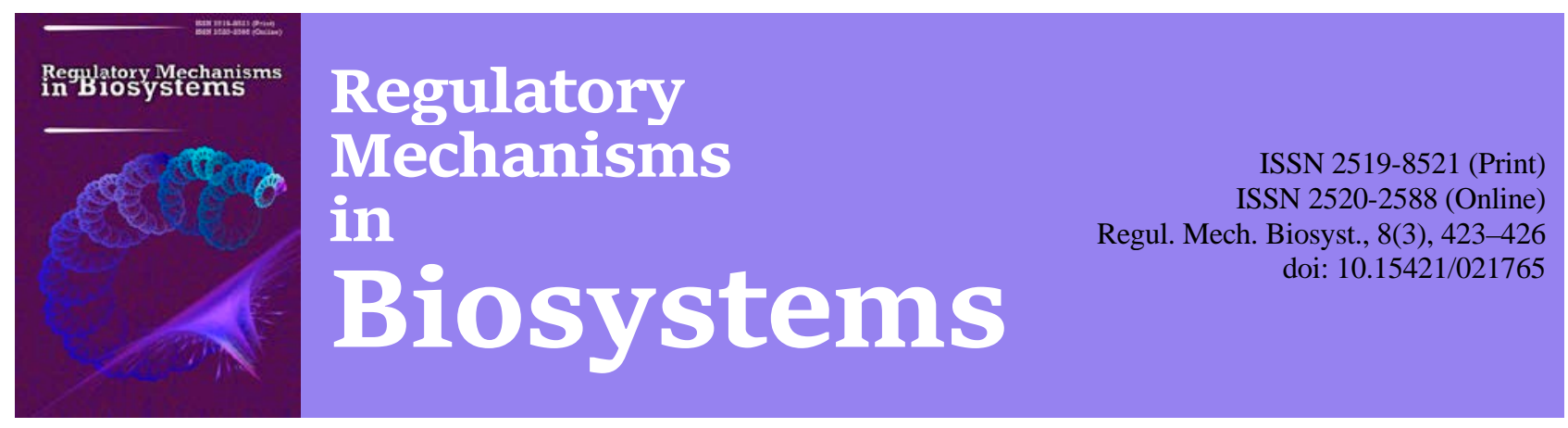

\title{
Dynamics of biochemical and immunological blood markers in patients with pseudoarthrosis of the femoral neck after total hip arthroplasty
}

\author{
S. E. Bondarenko*, M. Akonjom*, V. A. Filipenko*, D. V. Morozenko*, A. Badnaoui*, K. V. Gliebova** \\ *Sytenko Institute of Spine and Joint Pathology National Academy of Medical Science of Ukraine, Kharkiv, Ukraine \\ **National University of Pharmacy, Kharkiv, Ukraine
}

Article info

Received 14.07.2017

Received in revised form 09.08.2017

Accepted 13.08.2017

Sytenko Institute of Spine

and Joint Pathology of National

Ukrainian Academy of Medical

Sciences, Pushkinskaya Str., 80

Kharkiv, 61024, Ukraine.

Tel.: +38-067-574-01-51.

Email: bondarenke@gmail.com

National University of Pharmacy,

Pushkinska Str., 53,

Kharkiv, 61002, Ukraine

\begin{abstract}
Bondarenko, S. E., Akonjom, M., Filipenko, V. A., Morozenko, D. V., Badnaoui, A., \& Gliebova, K. V. (2017). Dynamics of biochemical and immunological blood markers in patients with pseudoarthrosis of the femoral neck after total hip arthroplasty. Regulatory Mechanisms in Biosystems, 8(3), 423-426. doi: 10.15421/021765
\end{abstract}

The importance of biomarkers to predict recovery following total hip arthroplasty (THA) is still unclear to clinicians. To assess the preoperative biomarkers of patients with pseudoarthrosis of the femoral neck and their dynamics in the early postoperative period after THA, 50 patients aged 33 to 82 years old, 18 males and 32 females diagnosed with pseudoarthrosis of the femoral neck after failed internal fixation and failed conservative management were studied. The control group consisted of 30 healthy people aged 27 to 50 years, 13 males, 17 females. Patients' blood was examined for biochemical markers upon admission, and then on the 7th and 14th days after surgery. Their blood serum content of total protein, albumin, glycoproteins, sialic acids, chondroitin sulfates, haptoglobin, glucose, cholesterol, triglycerides, ALT, AST, alkaline phosphatase, GGT, acid phosphatase, thymol index; interleukins (IL-1, IL-4 and IL-6.) and C-reactive protein was measured. The content of glycoproteins in the blood exceeded the norm by 2.3 times, chondroitin sulfate by 4.7 times, sialic acids by 1.5 times, haptoglobin by $55.8 \%$, fibrinogen by $19.1 \%$, globulin by $19,6 \%$, alkaline phosphatase activity by $72.3 \%$, IL-1 by 94.7 and IL- 6 by 3 times, C-reactive protein by 2.6 times. After THA there was a gradual decrease in blood biochemical and immunological markers. The most informative laboratory markers were glycoproteins, chondroitin sulfates, sialic acids, haptoglobin, activity of alkaline phosphatase, IL-1, IL-6 and IL-4, and C-reactive protein. Subsequent research is required to validate these dynamics.

Keywords: biomarkers; interleukins; glycoproteins; sialic acids; total hip replacement

\section{Introduction}

Pseudoarthrosis of the femoral neck is a common complication of femoral neck fracture following operative and conservative treatment (Dargan et al., 2012). Metabolic disorders in patients after THA may reveal themselves through changes in blood biochemical markers, including C-reactive protein, vitamins C, E and free radicals (Conway et al., 2015). Even the evaluation of the degree of invasiveness of surgery such as THA could be determined from the results of biochemical and immunological tests, especially creatine phosphokinase (CPK) and C-reactive protein (CRP); which are thought to be markers of muscle damage and post-operative inflammatory changes, respectively (Musil et al., 2008). It has been observed that in patients undergoing THA through a muscle sparing minimal invasive approach, activity of CPK and CRP was lower than in patients undergoing surgery following the standard procedure (Musil et al., 2008).

The use of laboratory diagnostic markers of hip pathology for the assessment of the condition of patients prior to THA; and to predict their recovery and functional outcome based on these markers still remains a prognostic challenge to clinicians (Yu et al., 2015; Wasko et al., 2015; Chen et al., 2016; Szypuła et al., 2016; Parvizi et al., 2016). So far, there is no clear list of hematological, immunological and biochemical tests for the initial assessment of health status of patients and monitoring the dynamics of their metabolic status following THA; much less the possibility of predicting their recovery. Some patients do well immediately post-op, and some take longer to fully recover. Whether the level of preoperative laboratory markers predicts the speed of postoperative recovery is yet to be ascertained. What could these markers and their levels possibly be?

Our study aims to assess the preoperative markers of patients with pseudoarthrosis of the femoral neck and their dynamics in the early postoperative period after THA. This may give clinicians a clue towards identifying prognostic information for recovery of patients.

\section{Materials and methods}

This study was conducted at the Department of Joint Pathology in conjunction with the department of Laboratory Diagnostics and Immunology of the Sytenko Institute of Spine and Joint Pathology of the National Academy of Medical Sciences of Ukraine. The period of the study covers 2011-2016. The study involved 50 patients aged 33 to 82 years old, 18 males and 32 females diagnosed with pseudoarthrosis of the femoral neck following failed internal fixation and failed conservative management requiring THA (Fig. 1, 2). The control group consisted of 30 healthy people aged 27 to 50 years, among them 13 males and 17 females.

Patients were excluded from this study if they did not have pseudoarthrosis due to prior hip fracture; were less than 20 years old, were known diabetics, known cancer patients, had known hepatic or renal dysfunction, and a history of hematological disorder or hypertension. 

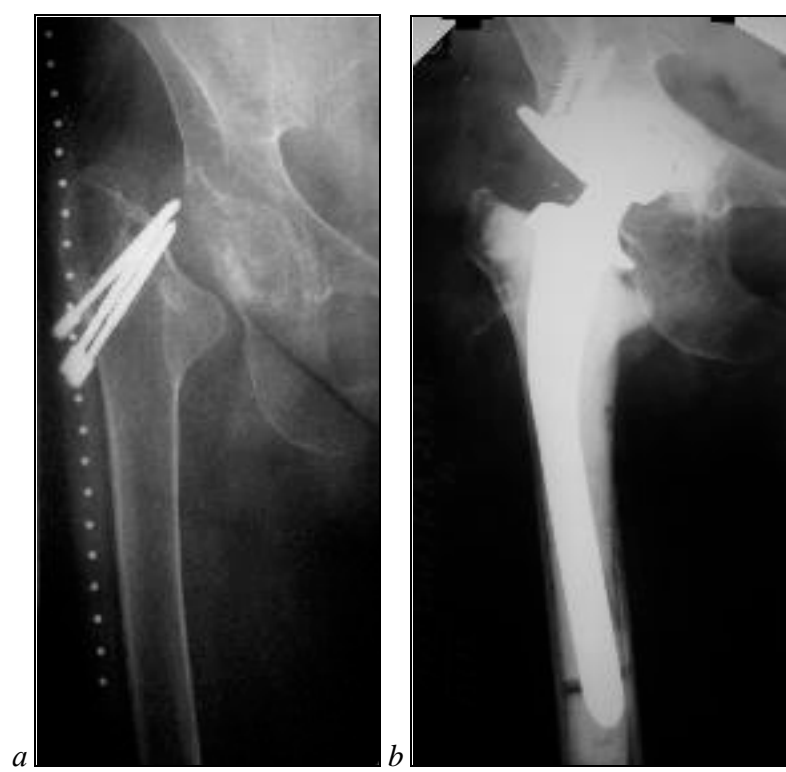

Fig. 1. Radiograph of a patient with pseudoarthrosis of the femoral neck following failed internal fixation: $a$ - preoperative, $b$ - postoperative radiograph after THA

The following markers were detected in the blood serum of patients: total protein by biuret test, albumin by reaction with bromocresol green, glycoproteins by the modified A. P. Stenberg and Y. N. Dotsenko method, sialic acids by the Hess method, chondroitin sulfate by the Nemeth-Csoka method modified by L. I. Slutsky, haptoglobin by reaction with rivanol, glucose by the enzymatic method. Total cholesterol content was determined by an enzymatic colorimetric method, triglyceride content was measured colorimetrically. The activity of the enzymes alanine aminotransferase (ALT), aspartate aminotransferase (AST), alkaline phosphatase and gamma-glutamyl transpeptidase (GGT) was determined by kinetic methods. The activity of acid phosphatase by the Bodansky method, the thymol test was performed following the method of Popper and Huergo. The content of fibrinogen in the blood plasma of pati- ents was determined using ready-made sets of reagents. Contents of interleukins (IL-1, IL-4, IL-6) and C-reactive protein in patients' blood serum was determined by solid-phase "sandwich" method a variation of immunoenzyme analysis. Measurements were made at a wavelength $\lambda=450 \mathrm{~nm}$ for all cytokines. Statistical analysis was performed using Statistica 10.0 software (Statsoft Inc., USA); by parametric Student criterion and non-parametric Wilcoxon criterion.
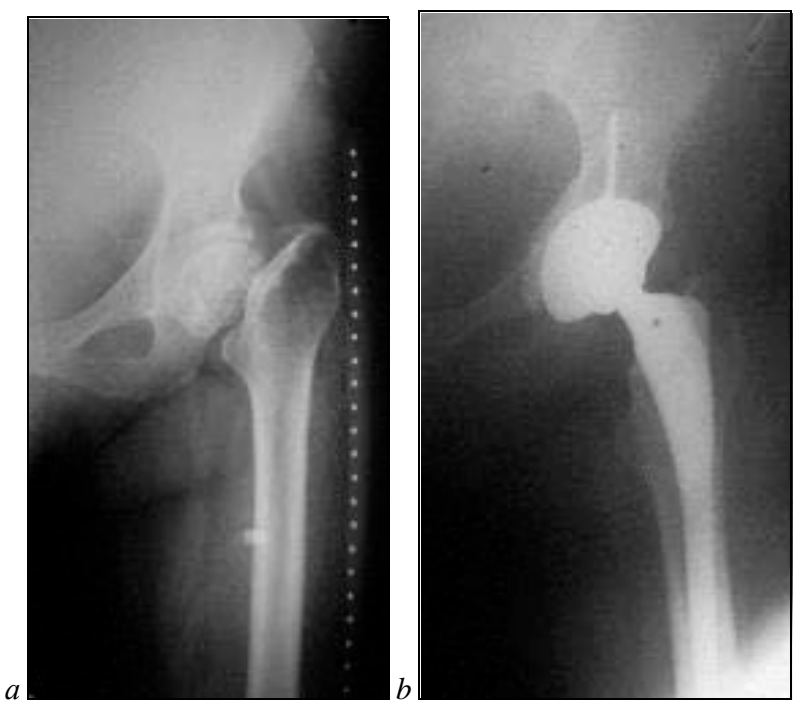

Fig. 2. Radiograph of a patient with pseudoarthrosis of the femoral neck following conservative management: $a$ - preoperative, $b$ - postoperative radiograph after THA

\section{Results}

In patients with pseudoarthrosis of the femoral neck, the glycolproteins content in the blood was increased by 2.3 times, chondroitin sulphates content by 4.7 times, sialic acids by 1.5 times compared with the control group (Table).

\section{Table}

Biochemical and immunological markers of the serum of patients with pseudoarthrosis of the femoral neck and compared with control group

\begin{tabular}{|c|c|c|c|c|c|}
\hline \multirow{2}{*}{$\begin{array}{l}\text { Biochemical and immulogical } \\
\text { markers }\end{array}$} & Control, $\mathrm{n}=30$ & Patients, $\mathrm{n}=50$ & Preceding the surgery & $\begin{array}{c}7 \text { days following } \\
\text { the surgery }\end{array}$ & $\begin{array}{c}14 \text { days following } \\
\text { the surgery }\end{array}$ \\
\hline & $\mathrm{M} \pm \mathrm{m}, \mathrm{Me}$ & $\mathrm{M} \pm \mathrm{m}$ & \multicolumn{3}{|c|}{ Me, \%25-\%75 } \\
\hline Glycoproteins, g/l & $0.57 \pm 0.01,0.57$ & $1.33 \pm 0.03^{* * *}$ & $1.33,1.14-1.44$ & $1.04,0.96-1.16$ & $0.79^{\diamond}, 0.73-0.90$ \\
\hline Chondroitin sulfates, g/l & $0.07 \pm 0.01,0.07$ & $0.33 \pm 0.01^{* * *}$ & $0.32,0.28-0.35$ & $0.25^{\diamond}, 0.22-0.28$ & $0.12^{\natural}, 0.11-0.14$ \\
\hline Sialic acids, $\mathrm{mmol} / \mathrm{l}$ & $2.05 \pm 0.05,1.97$ & $3.50 \pm 0.08^{* * *}$ & $3.40,3.10-4.04$ & $2.75^{\circ}, 2.29-3.04$ & $2.25^{\diamond}, 1.80-2.69$ \\
\hline Haptoglobin, g/l & $0.77 \pm 0.04,0.80$ & $1.20 \pm 0.03^{* * *}$ & $1.19,1.06-1.34$ & $0.86^{\diamond}, 0.80-0.94$ & $0.74^{\diamond}, 0.86-0.92$ \\
\hline Glucose, $\mathrm{mmol} / \mathrm{l}$ & $4.93 \pm 0.10,4.85$ & $4.70 \pm 0.07$ & $4.80,4.30-5.10$ & $4.74,4.28-5.10$ & $4.91,4.53-5.32$ \\
\hline Alkaline phosphatase, U/L & $157.07 \pm 8.05,151.50$ & $270.52 \pm 5.11^{* * *}$ & $278.00,251.50-290.00$ & $228.53^{\circ}, 201.54-247.86$ & $196.53^{\diamond}, 173.32-213.16$ \\
\hline Acid phosphatase, U/L & $3.90 \pm 0.16,4.00$ & $4.20 \pm 0.06$ & $4.10,4.00-4.30$ & $4.20,3.90-4.50$ & $4.22,3.90-4.50$ \\
\hline GGT, U/L & $27.43 \pm 1.81,31.50$ & $37.26 \pm 1.84 * *$ & $34.00,27.25-44.75$ & $31.96,27.16-42.86$ & $27.42,20.70-38.24$ \\
\hline Total cholesterol, mmol/l & $4.59 \pm 0.12,4.65$ & $4.73 \pm 0.04$ & $4.76,4.43-5.00$ & $4.70,4.48-5.04$ & $4.76,4.53-5.03$ \\
\hline Triglyceride, mmol/l & $1.12 \pm 0.05,1.12$ & $1.26 \pm 0.03$ & $1.23,1.12-1.43$ & $1.24,1.11-1.43$ & $1.27,1.12-1.45$ \\
\hline Thymol test, U. & $3.10 \pm 0.32,2.75$ & $4.69 \pm 0.24^{*}$ & $5.00,3.25-6.00$ & $4.35,2.83-5.22$ & $2.61^{8}, 1.96-3.35$ \\
\hline Fibrinogen, g/l & $2.33 \pm 0.10,2.40$ & $2.74 \pm 0.08^{*}$ & $2.79,2.20-3.28$ & $2.43,1.88-2.75$ & $1.80^{\circ}, 1.59-2.06$ \\
\hline Interleukin-1, pg/ml & $6.63 \pm 0.37,6.65$ & $12.91 \pm 0.47^{* * *}$ & $12.17,10.59-14.14$ & $8.54^{\circ}, 7.49-9.99$ & $7.42^{\circ}, 6.54-8.58$ \\
\hline Interleukin-6, pg/ml & $5.60 \pm 0.42,5.60$ & $16.60 \pm 0.48^{* * *}$ & $16.72,14.04-19.33$ & $14.82,12.29-17.28$ & $11.59^{\circ}, 9.15-13.33$ \\
\hline Interleukin-4, pg/ml & $7.48 \pm 0.57,8.05$ & $13.85 \pm 0.37 * * *$ & $13.45,12.15-15.66$ & $13.40,11.90-15.19$ & $9.52^{\diamond}, 8.28-11.56$ \\
\hline CRP, mg/l & $4.78 \pm 0.17,4.90$ & $12.50 \pm 0.47 * * *$ & $12.00,10.53-14.50$ & $8.28^{\circ}, 7.26-10.01$ & $5.80^{\circ}, 5.08-7.00$ \\
\hline
\end{tabular}

Notes: $*_{-} \mathrm{P}<0.05,{ }^{* *}-\mathrm{P}<0.01, * * *-\mathrm{P}<0.001$ compared with the control group; $\diamond-$ significance by Wilcoxon compared with the index before surgery.

Haptoglobin content in the blood was increased by $55.8 \%$, fibrinogen by $19.1 \%$, globulins by $19.6 \%$ as compared with the control group. Alkaline phosphatase activity as a marker of bone metabolism and disturbances was increased by $72.3 \%$, activity of acid phosphatase was not significantly changed (Table).

Activity of IL- 1 and IL-6 increased by 3 times, and by $94.7 \%$; and C-reactive protein increased by 2.6 times as indicators of acute inflammation alongside with increased IL-4 by $85.2 \%$ (Fig. 3). Also in this group of patients, there were increased liver enzyme activities probably due to uncontrolled intake of non-steroidal anti-inflammatory drugs as evidenced by increase in GGT activity by $36.1 \%$, as well as the presence of moderate hypoalbuminemia in the blood (Table). We were careful to exclude any systemic illness that could increase serum levels of these biomarkers. After THA, there was a 
decrease in the blood level of glycoproteins only on the 14th day. The decrease in content of chondroitin sulfate, sialic acids, haptoglobin, and alkaline phosphatase activity was gradual and was recorded on the 7th and 14th days of the post-operative period.
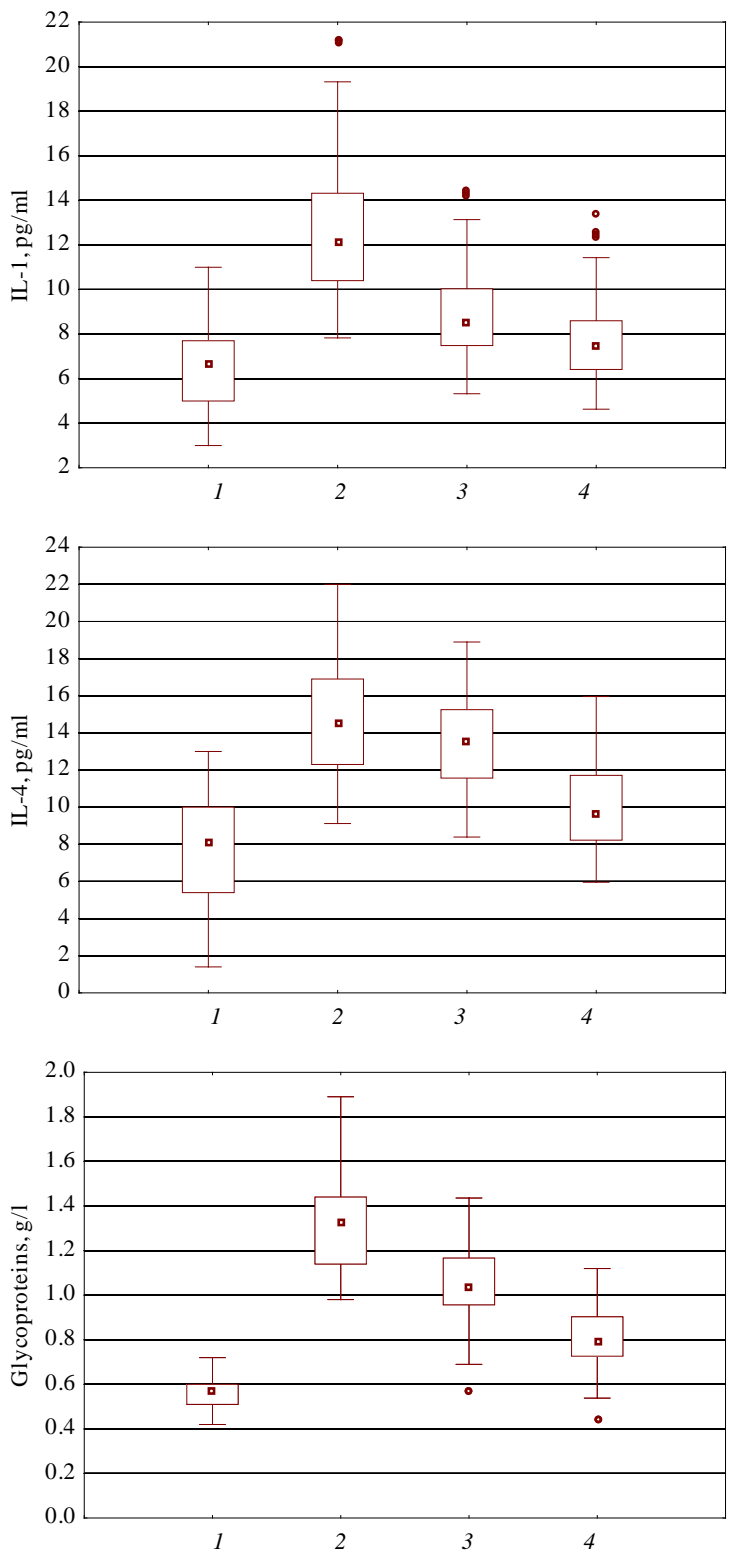

Thymol test, fibrinogen, IL-6 and IL-4 decreased in the blood only on the 14th day, and IL-1 and C-reactive protein content was decreased gradually from the 7th to the 14th days before reaching the level of the control group (Table and Fig. 3).
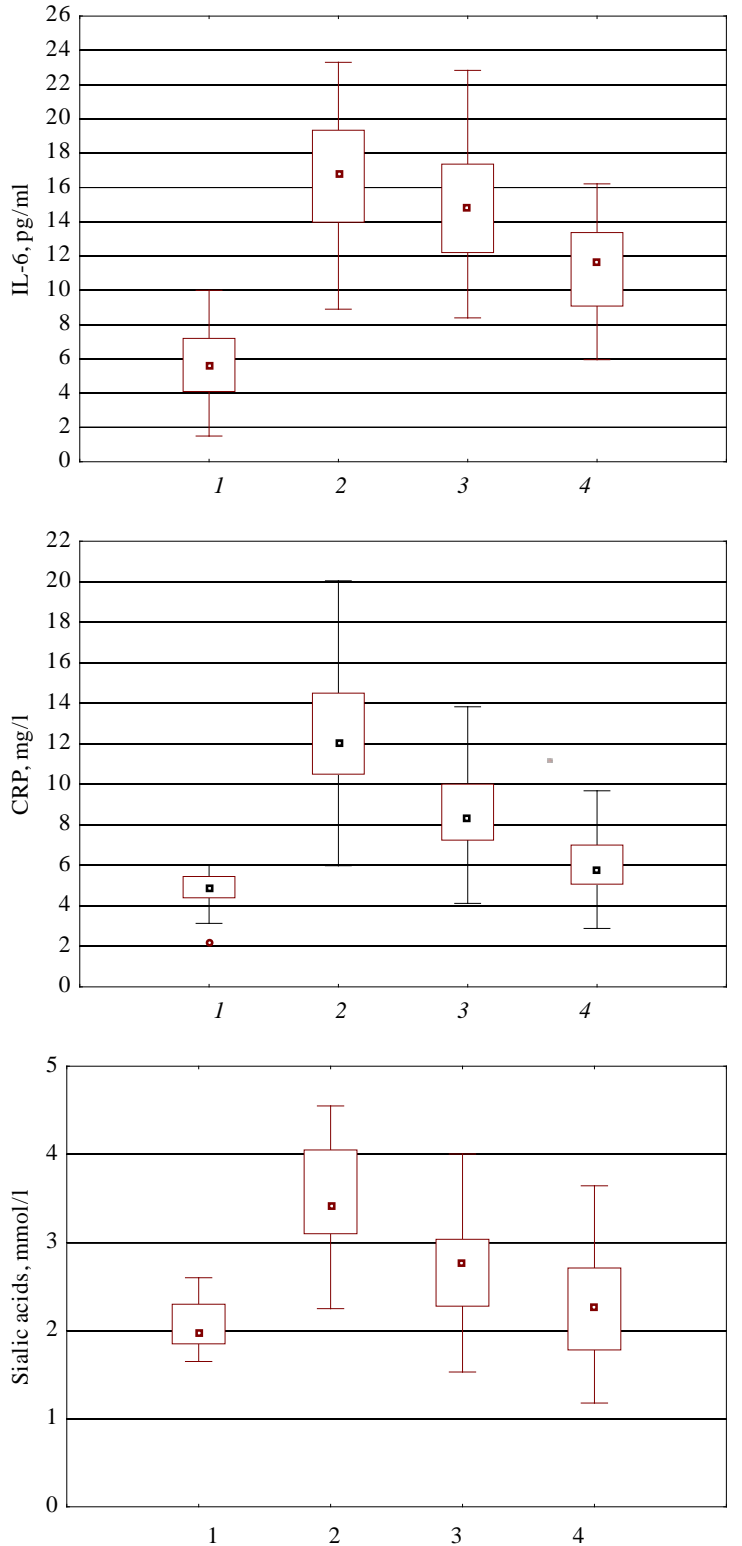

Fig. 3. The dynamics of biochemical and immunological markers in blood of patients with pseudoarthrosis of the femoral neck after total hip arthroplasty (Me): 1 - clinically healthy patients (controls), 2 - patients before surgery, 3 - 7 days post-operative, $4-14$ days post-operative (Table)

\section{Discussion}

Biochemical markers of bone turnover consist of proteins, enzymes, or molecules which are released into the circulation during formation or resorption and are measured in serum or in urine (Mark et al., 2015). The markers reflect enzymatic activities in the bone cells as measured by alkaline phosphatase level. The evaluation of biochemical and immunological markers is necessary considering that their release and clearance may be affected by other systemic factors which may complicate healing and prognosis (Singh et al., 2015). Moreover, biomarkers enable the physician to design a personalized treatment scheme which is individually tailored to the patient's need. However, widespread use of biomarkers has been limited due to the reported discrepancies that are mainly due to unclear biological function, variability and insufficient evidence of their prognostic value (Rissanen, 2013). The changes reflected in our results indicate pronounced inflammatory changes and subsequent attempts at healing in the body of patients with pseudoarthrosis of femoral neck following failed operative and conservative treatment. Typically, at the bone nonunion site, regenerative and inflammatory processes take place, this in the case of instability can trigger alternative changes in bone tissue leading to subsequent destruction (Mark et al., 2015). In the fracture zone the migration of neutrophils and macrophages occurs with secretion of a variety of cytokines, which in turn determine proliferation, angiogenesis and phagocytosis in the lesion (Mark et al., 2015). These processes are accompanied by a number of systemic hormonal changes that might provoke bone remodeling and subsequent resorption, involving osteoclasts. However, it should be noted that these morphological and biochemical changes in the body decrease in the 2-3 weeks after fracture. This may explain the lack of significant increase of acid phosphatase activity in patients' serum (Reikeras et al., 2014). The subsequent attempt at healing and formation of granulation tissue in the long-standing hip fracture site is subjected to mechanical strain leading to a false joint and 
instability, creating significant clinical disorders and impairment of function that require surgical treatment (Mark et al., 2015).

In patients undergoing THA due to pseudoarthrosis of the femoral neck, a decrease in inflammatory and biochemical markers according to the results of laboratory blood tests was observed (Drago et al., 2011; Reikeras et al., 2014). The study of biochemical and immunological markers of inflammation (interleukins, C-reactive protein), according to some authors, is essential for evaluating the degree of surgical trauma and identifying complications, mainly postoperative inflammation of periprosthetic tissue and periprosthetic infection (Drago et al., 2011; Reikeras et al., 2014). A common cause of revision THA is periprosthetic osteolysis (Eastwood et al., 2015). With the development of periprosthetic osteolysis in patients after failed THA, an increase in histochemical indicators of periprosthetic tissue responsible for the immune response to the implant implicates interleukins, mainly IL-1, IL-6 and IL-4 (Singh et al., 2015). It is also known that IL-6 is a marker for detecting persistent periprosthetic infection after primary as well as after revision THA (He et al., 2013; Hoell et al., 2015).

Another study showed that IL-6 is the most accurate laboratory marker for the diagnosis of periprosthetic infection compared with ESR, C-reactive protein and white blood cell count. IL-6 is an excellent screening test for the detection of patients with periprosthetic infection, with sensitivity of almost 100\% (Abou-El-Khier et al., 2013; Elgeidi et al., 2014). There is evidence that interleukin-level control in the blood of patients is important to evaluate their health condition and predict their postoperative recovery.

The content of IL-1, IL-6 and IL-10 in the serum of patients after the first day of THA was significantly higher than before the surgery ( $\mathrm{P}<0.001$ ), reached its peak levels on day 3 and subsequently decreased. However, the content of IL-2 in the serum of patients after surgery was significantly lower and gradually decreased to the level of standard (Mei et al., 2011). Furthermore, in obese patients with THA correlation between the level of IL-1 and IL-6 in the blood and body mass index was found. This signifies strengthening of the inflammatory response after surgery as a result of obesity complicating the surgery (Motaghedi et al., 2014).

Our study moved a step further to research not only the positive prognostic value of interleukins (IL-1, IL-4, IL-6) but also of other laboratory markers like glycoproteins, sialic acids, chondroitin sulfates, haptoglobin and fibrinogen, the dynamics of which show significant differences in the preoperative and postoperative periods ( $p<0.001$ ) (see Table I and Fig. 3-8). Reduction of these parameters on the 14th day after the operation, along with IL-1, IL-6 and IL-4, indicate a decrease in the activity of systemic inflammation in the bodies of patients. Analyzing the dynamics of these markers may prognosticate outcome and also predict the functional recovery of patients following THA. This is evidenced by the improvement of the clinical condition of patients after THA.

\section{Conclusion}

The most informative laboratory markers that could be suggested from our findings for assessing the condition of patients with pseudoarthrosis of the femoral neck before and after THA are C-reactive proteins; inflammatory cytokines IL-1, IL-6, anti-inflammatory cytokine IL-4, glycoproteins, chondroitin sulfates, sialic acids, haptoglobin, activity alkaline phosphatase $(\mathrm{p}<0.005)$. Gradual decrease in the biochemical markers on the 7th and 14th days after the operation indicates improvement in the condition of patients in the early postoperative period. Subsequent research is required to validate the dynamics of these markers in order to prognosticate outcome.

\section{References}

Abou-El-Khier, N. T., Ganainy, A.-R., Elgeidy, A., \& Rakha, S. A. (2013). Assessment of interleukin-6 and other inflammatory markers in the diagnosis of Egyptian patients with periprosthetic joint infection. Egyptian Journal of Immunology, 20(2), 93-99.

Berbari, E., Mabry, T., Tsaras, G., Spangehl, M., Erwin, P. J., Murad, M. H., Steckelberg, J., \& Osmon, D. (2010). Inflammatory blood laboratory levels as markers of prosthetic joint infection: A systematic review and meta-analysis. Journal of Bone and Joint Surgery. 1,92(11), 2102-2109.

Chen, X. X., Wang, T., Li, J., \& Kang, H. (2016). Relationship between inflammatory response and estimated complication rate after total hip arthroplasty. Chinese Medical Journal, 129(21), 2546-2551.

Conway, F. J., Talwar, D., \& McMillan, D. C. (2015). The relationship between acute changes in the systemic inflammatory response and plasma ascorbic acid, alpha-tocopherol and lipid peroxidation after elective hip arthroplasty. Clinical Nutrition, 34(4), 642-646.

Dargan, D., McCaffrey, D., \& Kealey, W. D. C. (2012). Pseudoarthrosis of femoral neck stress fracture treated with open reduction, sliding hip screw and bone morphogenic protein. International Journal of Surgery Case Reports, 3(11), 529-532.

Drago, L., Vassena, C., Dozio, E., Corsi, M. M., De Vecchi, E., Mattina, R., \& Romanò, C. (2011). Procalcitonin, C-reactive protein, interleukin-6, and soluble intercellular adhesion molecule-1 as markers of postoperative orthopaedic joint prosthesis infections. International Journal of Immunopathology and Pharmacology, 24(2), 433-340.

Eastwood, S. E., John, A., Jones, S. A., Hodgson, H., Mason, D., Waddington, R., \& Wei, X. (2015). Osteoclastogenesis-related cytokines and peri-prosthetic osteolysis in revision metal-on-metal total hip replacements. Hip International, 25(4), 355-360.

Elgeidi, A., Elganainy, A. E., Abou Elkhier, N., \& Rakha, S. (2014). Interleukin-6 and other inflammatory markers in diagnosis of periprosthetic joint infection. International Orthopaedics, 38(12), 2591-2595.

Glehr, M., Friesenbichler, J., Hofmann, G., \& Bernhardt, G. A. (2013). Novel biomarkers to detect infection in revision hip and knee arthroplasties. Clinical Orthopaedics and Related Research, 471(8), 2621-2628.

He, T., Wu, W., Huang, Y., Zhang, X., Tang, T., \& Dai, K. (2013). Multiple biomarkers analysis for the early detection of prosthetic aseptic loosening of hip arthroplasty. International Orthopaedics, 37(6), 1025-1031.

Hoell, S., Borgers, L., Gosheger, G., Dieckmann, R., Schulz, D., Gerss, J., \& Hardes, J. (2015). Interleukin-6 in two-stage revision arthroplasty: What is the threshold value to exclude persistent infection before re-implantation? Bone and Joint Journal, 97B(1), 71-75.

Mei, X. L., Guo, T., \& Zhao, J. N. (2011). Clinical significance and expression of the inflammatory cytokines (IL-1, IL-2, IL-6 and IL-10) in blood serum of the patients after total hip replacement. China Journal of Orthopaedics and Traumatology, 24(6), 463-465.

Motaghedi, R., Bae, J. J., Memtsoudis, S. G., \& Kim, D. H. (2014). Association of obesity with inflammation and pain after total hip arthroplasty. Clinical Orthopaedics and Related Research, 472(5), 1442-1448.

Musil, D., Stehlík, J., \& Verner, M. (2008) A comparison of operative invasiveness in minimally invasive anterolateral hip replacement (MIS-AL) and standard hip procedure, using biochemical markers. Acta Chirurgiae Orthopaedicae et Traumatologiae Cechoslovaca, 75(1), 16-20.

Parvizi, J., Fassihi, S. C., \& Enayatollahi, M. A. (2016). Diagnosis of periprosthetic joint infection following hip and knee arthroplasty. Orthopedic Clinics of North America, 47(3), 505-515.

Reikeras, O., Borgen, P., Reseland, J. E., \& Lyngstadaas, S. P. (2014). Changes in serum cytokines in response to musculoskeletal surgical trauma. BMC Research Notes, 7, 7.

Rissanen, J. (2013) Markers of bone turnover in preclinical development of drugs for skeletal diseases. Department of Cell Biology and Anatomy, University of Turku Doctoral Programme, Finland, p. 13-14.

Singh, G., Nuechtem, J. V., Meyer, H., Fiedler, G. M., Awiszus, F., Junk-Jantsch, S., Bruegel, M., Pflueger, G., \& Lohmann, C. H. (2015). Particle characterisation and cytokine expression in failed small-diameter metal-on-metal total hip arthroplasties. Journal of Bone and Joint Surgery, 97B(7), 917-923.

Szypuła, J., Cabak, A., Kiljański, M., Boguszewski, D., \& Tomaszewski, W. (2016). Comparison of biocompatibility of cemented vs. cementless hip joint endoprostheses based on postoperative evaluation of proinflammatory cytokine levels. Medical Science Monitor, 22, 4830-4835.

Wasko, M. K., Bobecka-Wesołowska, K., Tomasiuk, R., \& Kowalczewski, J. (2015). Measurement of the inflammatory response in the early postoperative period after hip and knee arthroplasty. Clinical Chemistry and Laboratory Medicine, 53(11), 1785-1792.

Yu, D., Yin, Z., Fu, C., \& Yu, R. (2014). Impact on red blood cell immunity patterns in postoperative phase following total hip arthroplasty. CentralEuropean Journal of Immunology, 39(3), 377-383. 